\title{
The impact on patient flow after the integration of nurse practitioners and physician assistants in 6 Ontario emergency departments
}

\author{
James Ducharme, MD; ${ }^{*}$ Robert J. Alder, $\mathrm{PhD}{ }^{\dagger}$ Cindy Pelletier, $\mathrm{MSc} ;{ }^{\ddagger}$ Don Murray, MSc; ${ }^{\S}$ \\ Joshua Tepper, MD, MPHף
}

\section{ABSTRACT}

Objective: We sought to assess the impact of the integration of the new roles of primary health care nurse practitioners (NPs) and physician assistants (PAs) on patient flow, wait times and proportions of patients who left without being seen in 6 Ontario emergency departments (EDs).

Methods: We performed a retrospective review of health records data on patient arrival time, time of initial assessment by a physician, time of discharge from the ED and discharge status.

Results: Whether a PA or NP was directly involved in the care of patients or indirectly involved by being on duty, the wait times, lengths of stay and proportion of patients who left without being seen were significantly reduced. When a PA or NP were directly involved in patients' care, patients were 1.6 (95\% confidence interval $[\mathrm{Cl}] 1.3-2.1, p<0.05)$ and $2.1(95 \% \mathrm{Cl}$ $1.6-2.8, p<0.05)$ times more likely to be seen within the wait time benchmarks, respectively. Lengths of stay were $30.3 \%$ (95\% Cl 21.6\%-39.0\%, $p<0.01$ ) and $48.8 \%$ (95\% Cl $35.0 \%-$ $62.7 \%, p<0.01$ ) lower when PAs and NPs, respectively, were involved. When PAs and NPs were not on duty, the proportion of patients who left without being seen were $44 \%(95 \% \mathrm{Cl}$ $31 \%-63 \%, p<0.01)$ and $71 \%(95 \% \mathrm{Cl} 53 \%-96 \%, p<0.05)$, respectively.

Conclusion: The addition of PAs or NPs to the ED team can improve patient flow in medium-sized community hospital EDs. Given the ongoing shortage of physicians, use of alternative health care providers should be considered. These results require validation, as their generalizability to other locations or types of EDs is not known.

Keywords: emergency department, physician assistant, nurse practitioner, wait times, length of stay
RÉSUMÉ

Objectif : Nous avons cherché à évaluer l'impact de l'intégration des nouveaux rôles des infirmières praticiennes (IP) et des adjoints aux médecins (AM) en contexte de soins primaires sur le cheminement des patients, les temps d'attente et le pourcentage de patients qui ont quitté l'urgence sans avoir été vus par un médecin dans 6 services d'urgence en Ontario.

Méthodes : Nous avons procédé à un examen rétrospectif de données dans les dossiers de santé des patients sur l'heure d'arrivée à l'urgence, I'heure de l'évaluation initiale par un médecin, I'heure du congé et l'état à la sortie de l'urgence.

Résultats : Que les AM ou les IP aient participé directement aux soins du patient ou indirectement, en étant de service, les temps d'attente, la durée du séjour et le pourcentage des patients qui ont quitté l'urgence sans avoir été vus par un médecin ont été considérablement réduits. Quand les AM ou les IP participaient directement aux soins des patients, ces derniers étaient respectivement 1,6 fois (intervalle de confiance $[I C]$ à $95 \%$ de 1,3 à $2,1, p<0,05$ ) et 2,1 fois (IC à $95 \%$ de $1,6$ à $2,8, p<0,05)$ plus susceptibles d'être vus par un médecin dans les délais fixés pour les temps d'attente. La durée de séjour était respectivement 30,3\% (IC à $95 \%$ de 21,6 à $39,0 \%, p<0,01$ ) et $48,8 \%$ (IC à $95 \%, 35,0$ à $62,7 \%, p<0,01$ ) plus faible lorsque les AM et les IP participaient aux soins. Lorsque les AM ou les IP n'étaient pas de service, le pourcentage de patients qui ont quitté l'urgence sans avoir été vus par un médecin était respectivement de $44 \%$ (IC à $95 \%$ de 31 à $63 \%, p<0,01$ ) et de $71 \%$ (IC à $95 \%$ de 53 à $96 \%, p<0,05$ ).

Conclusion : L'ajout d'AM et d'IP à l'équipe du service d'urgence peut améliorer le cheminement des patients dans les urgences des hôpitaux communautaires de taille moyenne. Compte tenu de la pénurie actuelle de médecins, le recours à d'autres professionnels de la santé devrait être envisagé. Ces constatations doivent être validées, car leur généralisabilité à d'autres régions ou types de services d'urgence n'est pas connue.

\footnotetext{
From the *Department of Medicine, McMaster University, Hamilton, Ont., the TFaculty of Medicine and Dentistry, University of Western Ontario, London, Ont., ¥MedEmerg International Ltd., Mississauga, Ont., §Harry Cummings and Associates, Guelph, Ont., and the IDepartment of Family and Community Medicine, University of Toronto, Toronto, Ont.
}

Submitted Jul. 8, 2008; Revised Mar. 2, 2009; Accepted Mar. 11, 2009

This article has been peer reviewed.

CJEM 2009;11(5):455-61 


\section{INTRODUCTION}

Wait times for health care in Ontario have become a major concern and the focus of numerous provincial initiatives. Emergency department (ED) wait times are a focal point for these initiatives. From 2003 to 2004, there were 4364000 patient visits to Ontario EDs. ${ }^{1}$ Of these, approximately one-half involved a wait of less than 1 hour to be seen by a physician. For $10 \%$ of patients, this wait was less than 10 minutes; another $10 \%$ waited 165 minutes or longer. ${ }^{1}$ Time to physician varied by assigned acuity level. Patients who were triaged to level I, the most acute Canadian Emergency Department Triage and Acuity Scale (CTAS) score, had a median wait of 5 minutes. Patients who were triaged to levels II, III, IV and V had median wait times of approximately 30, 60, 50 and 40 minutes, respectively. ${ }^{1}$ Many of the more acutely ill patients (CTAS I, II and III) are not seen within the operating objectives (benchmarks) of the CTAS. ${ }^{2}$

Delays in assessment and care may have negative effects on patient care and outcomes. Long wait times could potentially result in worse patient outcomes, greater patient suffering, patient dissatisfaction, more difficulty retaining and recruiting staff, a higher risk of infectious disease outbreaks and an increased risk of medical errors. ${ }^{3}$ The risk of violence directed toward hospital staff and physicians may also be higher in environments with significant delays. Moreover, in a negative work environment, ED staff may be less productive and less able to effectively teach or perform research. ${ }^{3}$

Prolonged wait times, often exacerbated at peak hours, may lead to patients leaving the ED before being assessed by a physician. In Ontario, in 2003 and 2004, 136000 patients $(3.1 \%)$ visiting the ED left without being seen (LWBS). ${ }^{1}$ As numbers of patients in the waiting room increase, so too does the proportion of patients who leave without being seen by a physician. ${ }^{4}$ Although ambulatory patients do not directly contribute to overcrowding, waiting room numbers are considered an integral variable when considering overcrowding. ${ }^{5}$ Many of those who leave have no access to primary health care or have conditions that require immediate attention or follow-up care.

High LWBS rates and lengthy wait times are a result of a variety of factors, one of which is a shortage of physicians and nurses. ${ }^{6}$ As part of the Ontario government's Emergency Department Action Plan, the roles of primary health care nurse practitioners (NPs) and physician assistants (PAs) were introduced in 6 medium-sized community hospital EDs. The purpose of this study was to examine the impact of PAs and NPs in EDs, focusing on patient wait times, lengths of stay (LOS) and LWBS rates. We hypothesized that the addition of these new roles would reduce wait times, LOS and LWBS rates.

\section{METHODS}

Data for this study were collected as part of a comprehensive project to evaluate the integration of the new roles within EDs. This evaluation included an analysis of administrative changes, team effectiveness surveys, team development sessions with focus groups, and health records data pertaining to patient care and flow. The health record data component is the focus of this analysis.

\section{Overview of the project}

In 2006, the Ontario government announced the launch of HealthForceOntario (HFO), a health human resources strategy. In its new roles component, HFO supports interprofessional care by introducing new personnel to collaborative health care teams to help reduce wait times for high-demand services, such as emergency care, surgical services and cancer care. Subsequently, 6 interested community hospitals with ED volumes varying from 23770 to 66136 patient visits per year (in 2005 and 2006), were selected by the Ministry of Health and Long-Term Care to expand their ED team to include PAs, primary health care NPs and acute care nurse specialists (AC NS). As only one AC NS was recruited, the role was excluded from analysis in this study. A summary of patient volumes and personnel in each of the 6 study sites is provided in Table 1 . Although 2 sites had occasional learners on elective rotations, none of the sites were teaching hospitals with

\begin{tabular}{|c|c|c|c|c|c|}
\hline Hospital & $\begin{array}{c}\text { ED } \\
\text { volume } \\
\text { (approx.) }\end{array}$ & $\begin{array}{l}\text { Full-time } \\
\text { equivalent } \\
\text { physicians }\end{array}$ & PA & NP & AC NS \\
\hline A & 24750 & 7 & Yes & Yes & No \\
\hline B & 28000 & 4 & Yes & Yes & No \\
\hline C & 37500 & 7 & Yes & No & No \\
\hline D & 38000 & 10 & Yes & Yes & No \\
\hline E & 44250 & 11.5 & Yes & Yes & Yes \\
\hline $\mathrm{F}$ & 60500 & 12 & No & Yes & No \\
\hline
\end{tabular}


regularly scheduled trainees. The new staff members were expected to function as additions to existing physician staff, not replacements. They were to work shifts covering the predetermined busiest periods for each ED. For all sites, this resulted in extra coverage between 10 am and $10 \mathrm{pm}$. Since some sites only had one worker, it was not possible to ensure coverage 7 days per week. As a result, each site had periods of time with no extra coverage that could act as control periods for comparative measurements related to this project.

Physician assistants were introduced to Ontario through this project as an unregulated provider, ${ }^{7}$ and work without medical directives under the supervision of a registered physician who was responsible for all patient care. Unlike NPs, PAs are precluded from taking independent medical actions. The specific duties of each PA varied by site, but, in general, PAs saw patients with a wider range of acuity levels than NPs. Primary health care NPs are regulated health professionals registered with the College of Nurses of Ontario (CNO). As per CNO's directives, NPs practise autonomously for CTAS-IV and $-\mathrm{V}$ patients and work in conjunction with a physician to see CTAS-III or higher acuity patients. ${ }^{8}$ Physicians interacted with both NPs and PAs for interpreting any diagnostic imaging and for the management of CTAS-III or higher acuity patients. All members of the new staff were expected to comply with ED policies and guidelines. In some centres with higher volumes, an NP and PA occasionally worked simultaneously, although this was uncommon.

At all sites, integration of the new workers occurred in conjunction with a full-day session that discussed the roles and permitted activities of the NPs and PAs, and involved team-building discussions and small-group sessions to identify local problems and possible solutions.

\section{Data collection}

Each participating hospital provided the required data from their administrative health records and the standardized data that is regularly submitted according to provincial reporting requirements. These reporting requirements and the associated collection protocols were not modified during the study. Data collection occurred during 2 periods: Nov. 13, 2006, to Dec. 3, 2006 (baseline) and Jun. 11, 2007, to Jun. 29, 2007 (6-mo postimplementation follow-up). The data collection throughout these 2 periods took place during a 14-day interval; some hospitals started and ended a few days sooner than others.
The following information was obtained for every ED patient during the study periods for each of the 6 sites:

- date of patient visit

- time of triage

- type of physician involved in patient treatment (emergency physician, family physician, etc.)

- type of enhanced provider (PA, NP) involved in patient treatment

- CTAS score

- wait time (triage to initial assessment by physician)

- length of stay in ED (triage to disposition)

- discharge disposition

Personal identifiers, such as name and age, were not included in the spreadsheet to protect the confidentiality of the patients.

Health records data were analyzed to assess both the direct and indirect impact of the presence of the NP or PA. This was done by measuring time intervals for patients treated directly by an NP or PA, as well as by comparing time data between patients who were and were not cared for while an NP or PA was on duty. The impact of the new providers on wait times, LOS and proportion of LWBS between baseline and follow-up was measured using multivariate analysis (logistic regression or analysis of covariance, depending on whether the variable was dichotomous or continuous, respectively) with adjustment for hospital, time of patient visit and acuity level. The analyses were performed using SPSS 15.0 (SPSS Inc.).

We based the determination of whether a provider was involved in patient care on the health records. After adjustment, wait times and LOS were compared between patients with a PA and/or NP directly involved in their care and patients treated without additional workers present in the department.

Similarly, patients' wait times and LOS were analyzed for the possible indirect effect of the PA or NP being on duty, but not directly involved in those patients' care. Wait times and LOS of patients at the time of registration were compared when the PA or NP was on duty versus when a PA or NP was not on duty. Patients who had a PA or an NP involved in their care were excluded to isolate those who would have only indirectly benefited from the provider being on duty.

For wait times (i.e., triage to initial assessment by a physician), we analyzed the data according to whether a patient's wait times met the acuity-related benchmark set by the Canadian Association of Emergency Physicians, National Emergency Nurses Affiliation and the Association des médecins d'urgence du Québec. ${ }^{2}$ The 
ethics review boards of all involved hospitals approved the release of the data for the purposes of this study.

\section{RESULTS}

Data on 19592 patient visits were collected during the 2 periods of data collection. Of those, 9585 (48.9\%) visits took place during the baseline period and 10007 $(51.1 \%)$ occurred during the follow-up period. The distribution of visits by level of acuity is outlined in Table 2. The PAs were on duty for 1076 visits and were directly involved in patient care for 396 of those visits.

\begin{tabular}{|c|c|}
\hline \multicolumn{2}{|c|}{$\begin{array}{l}\text { Table 2. Distribution of patient visits, by } \\
\text { acuity level }\end{array}$} \\
\hline CTAS level & No. (\%) of visits \\
\hline I & $\begin{array}{ll}76 & (0.4)\end{array}$ \\
\hline II & $2736 \quad(14.0)$ \\
\hline III & $7893 \quad$ (40.3) \\
\hline IV & $6810 \quad$ (34.8) \\
\hline V & $1205 \quad(6.2)$ \\
\hline Missing & 872 \\
\hline Total & $19592(100.0)$ \\
\hline
\end{tabular}

Table 3. Proportion of patient visits for which wait time benchmarks were met, by acuity and physician assistant involvement

\begin{tabular}{lccc} 
& \multicolumn{2}{c}{ PA involvement, $\%$} & \\
\cline { 2 - 3 } CTAS level & No & Yes & Difference, \% \\
\hline II & 12.2 & 18.2 & 6.0 \\
III & 13.5 & 12.1 & -1.4 \\
IV & 29.8 & 38.5 & 8.6 \\
V & 50.2 & 72.2 & 22.0 \\
Total & 21.4 & 28.3 & 6.8 \\
\hline CTAS = Canadian Emergency Department & Triage and Acuity Scale; PA = physician \\
assistant. &
\end{tabular}

Table 4. Proportion of patient visits for which wait time benchmarks were met, by acuity and nurse practitioner involvement

\begin{tabular}{lccc} 
& \multicolumn{2}{c}{ NP involvement, $\%$} & \\
\cline { 2 - 3 } CTAS level & No & Yes & Difference, \% \\
III & 17.7 & 26.7 & 8.9 \\
IV & 33.1 & 53.8 & 20.7 \\
V & 64.9 & 91.7 & 26.8 \\
Total & 25.0 & 52.6 & 27.6 \\
\hline $\begin{array}{l}\text { CTAS = Canadian } \\
\text { practitioner. }\end{array}$ &
\end{tabular}

The NPs were on duty for 1744 visits and were directly involved in patient care for 298 of those visits. Table 3 and Table 4 depict the absolute changes in the proportion of patients who were seen within the benchmarks with PA and NP involvement, respectively. After adjustment for hospitals, time of day and acuity, when a PA was involved in patient care the odds of the patient being seen within the benchmark wait time was 1.6 times greater than when the PA was not involved (95\% confidence interval [C] $1.3-2.1, p<0.05$ ). When the NP was involved, the odds were 2.1 times greater (95\% CI 1.6-2.8, $p<0.05$ ).

A similar analysis was performed regarding the effect of an NP or PA being on duty, but not being directly involved with the patients' care, and the odds ratios were also positive. Table 5 and Table 6 show the absolute improvements in the proportion of patients who were seen within the benchmarks during these scenarios. After adjustment, when a PA was on duty, the odds of a patient being assessed within the wait time benchmark were 1.9 times higher than when a PA was not on duty (95\% CI 1.6-2.4, $p<0.01$ ). When an NP was working, the odds were 1.5 times higher $(95 \%$ CI $1.3-1.8, p<0.01)$.

Table 5. Proportion of patient visits for which wait time benchmarks were met, by acuity and on-duty status of physician assistant

\begin{tabular}{lccc} 
& \multicolumn{2}{c}{ PA on duty, $\%$} & \\
\cline { 2 - 3 } CTAS level & No & Yes & Difference, \% \\
\hline II & 11.8 & 13.5 & 1.7 \\
III & 11.6 & 12.7 & 1.1 \\
IV & 26.8 & 34.8 & 8.0 \\
V & 57.3 & 60.3 & 3.0 \\
Total & 18.5 & 23.8 & 5.3 \\
\hline CTAS = Canadian & Emergency Department & Triage and Acuity Scale; PA = physician \\
assistant. &
\end{tabular}

Table 6. Proportion of patient visits for which wait time benchmarks were met, by acuity and on-duty status of nurse practitioner

\begin{tabular}{lccc} 
& \multicolumn{2}{c}{ NP on duty, \% } & \\
\cline { 2 - 3 } CTAS level & No & Yes & Difference, \% \\
\hline II & 13.6 & 12.3 & -1.3 \\
III & 15.5 & 15.1 & -0.4 \\
IV & 30.3 & 35.1 & 4.8 \\
V & 64.2 & 70.7 & 6.5 \\
Total & 22.0 & 25.3 & 3.3 \\
\hline CTAS = Canadian Emergency Department Triage and Acuity Scale; NP = nurse \\
practitioner.
\end{tabular}


For LOS, in addition to adjusting for hospital, time of day and acuity, we also compared the data between patients with a PA or NP directly involved in their care and patients seen by physicians when an NP or PA was not present (Fig. 1). When a PA was involved in patient care, the length of stay in the ED was $30.3 \%$ shorter than when a PA was not present (95\% CI $21.6 \%-39 \%$, $p<0.01)$. The mean LOS changed from 262.4 minutes to 182.9 minutes when a PA was involved. When an NP was involved, the mean LOS was $48.8 \%$ shorter $(95 \%$ CI $35 \%-62.7 \%, p<0.01$ ), dropping from $256.3 \mathrm{~min}-$ utes to 131.1 minutes.

Although not as dramatic an effect, having a PA or $\mathrm{NP}$ on duty also had a statistically significant effect (PA: 95\% CI 3.6\%-14.1\%, $p<0.01$; NP: 95\% CI 4.6\%$13.9 \%, p<0.01$ ) on the mean LOS of patients (Fig. 2). For PAs, the mean decreased from 304.2 minutes to 277.2 (8.9\% shorter, 95\% CI 3.6\%-14.1\%, $p<0.01$ ), and, for NPs, the mean dropped from 257.7 minutes to 233.81 (9.3\% shorter, 95\% CI 4.6\%-13.9\%, $p<0.01)$ when they were on duty.

When a PA or an NP was on duty, the proportion of patients who left without being seen was significantly reduced. The absolute improvements, not controlling for hospital or acuity, were $24.6 \%$ for PAs (the LWBS rate decreased from $6.5 \%$ to $4.9 \%$ ) and $17.6 \%$ for NPs (the LWBS rate dropped from $5.1 \%$ to $4.2 \%$ ). When a PA was on duty, controlling for hospital, time of day and acuity, the likelihood that a patient left without being seen was less than half than when a PA was not on duty $(44 \%, 95 \%$ CI $31 \%-63 \%, p<0.01)$. With an NP on duty there was a $29 \%$ reduction in LWBS rates (95\% CI $4 \%-47 \%, p<0.05)$ compared with those when an NP was not on duty.

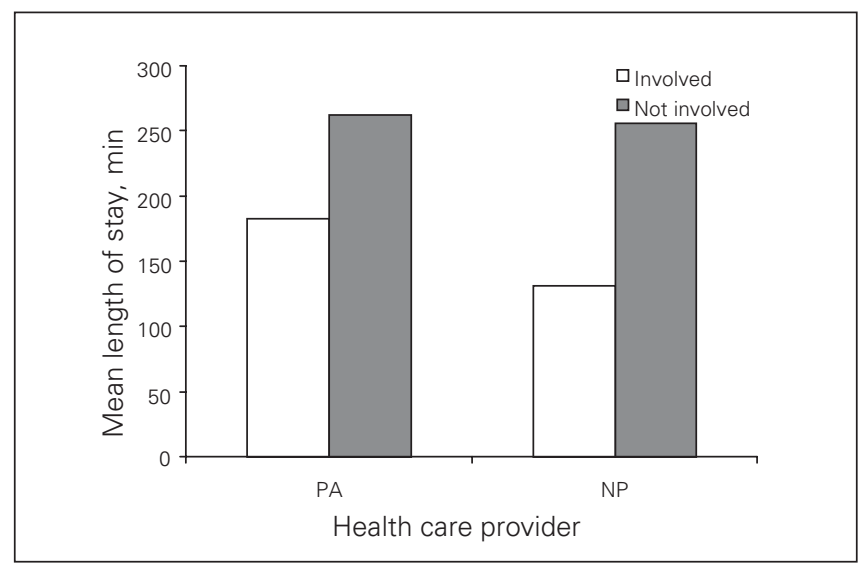

Fig. 1. Mean length of stay in patients with nurse practitioner (NP) or physician assistant (PA) involved versus not involved, Ontario emergency departments, 2007.

\section{DISCUSSION}

In what we believe to be the first Canadian study to analyze the impact of NPs and PAs on patient flow in the $\mathrm{ED}$, the integration of these health care roles was associated with reductions in wait times, LOS in the ED and proportions of patients who left without being seen. Past studies in British, US, Australian and Spanish settings have found similar positive results. ${ }^{9-14}$ Additionally, a recent Canadian study found that patients are very satisfied with the care they receive from providers other than physicians. ${ }^{15}$ It would therefore seem that integrating alternate health care providers into EDs in Canada is a viable option. All but the latter study took place in health care systems outside Canada, and the NPs or PAs were not fully integrated into the ED team but rather in a fasttrack unit or working independently of the ED team.

We saw a direct effect on patient flow, presumably because the addition of primary health care providers increases the number of workers able to assess and treat patients. Flow of patients can thus be improved within the ED without establishing a separate fast-track area. An additional indirect effect is likely that physicians can focus on the more ill and injured, knowing that those of lesser acuity will, at least initially, be seen by the PA or NP. As discussed earlier, in the Canadian context there is a shortage of physicians and other staff in EDs and there is regular ED overcrowding. ${ }^{3}$ Staff shortages can lead to delays in patient care, which in turn may lead to a lower quality of care, greater morbidity and mortality, and violence aimed at hospital staff., ${ }^{3,16}$

Various initiatives have been developed to meet the increasing demands on health care systems and organizations to provide more timely services with finite

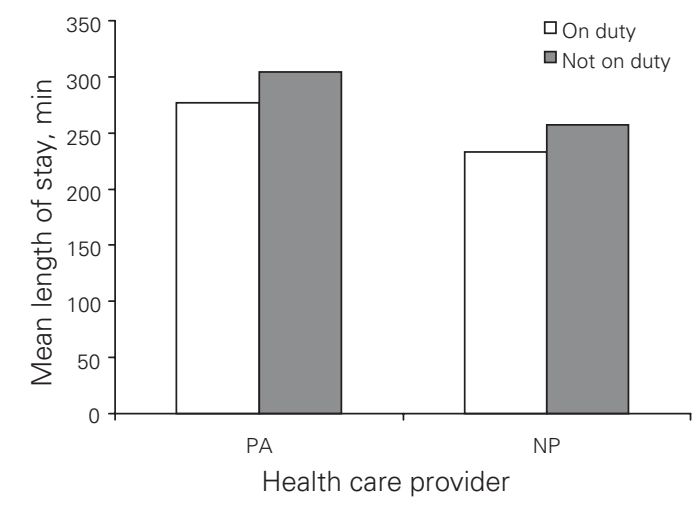

Fig. 2. Mean length of stay for patient visits with a physician assistant (PA) or nurse practitioner (NP) on duty versus not on duty, Ontario emergency departments, 2007. 
human and financial resources. These include human resource mix initiatives (such as increasing support staff and developing rapid response teams) and human resource supply initiatives (such as increasing educational seats and increasing recruitment). New ways of delivering care are required, and the expansion of interprofessional teams may be one such approach. Our findings support the integration of NPs and PAs as primary health care providers into Canadian EDs. The reductions we found in wait times and LOS suggest that the presence of the new roles can help to improve the efficiency of ED patient care. However, as more patients are assessed, it may well be that such an increase in front-end efficiency may overwhelm other areas already working at peak capacity. For example, nurses and laboratory staff might not be able to keep up with the greater number of orders written up during busy periods. The ability to see increasing numbers could rapidly be offset if the system does not solve the current ED exit block for admitted patients. At many sites where support services, such as diagnostic imaging, provide single-team coverage, such services may not be able to meet the increased demand that would be seen with more primary providers. Initiatives involving NPs and/or PAs require not only buy-in by all ED team members, but system planning and anticipation to succeed. Careful analysis of the impact of additional providers will be needed to ensure that necessary modifications are made if this new model is adopted.

An equally if not more important question to ask is whether the quality of patient care will suffer in the effort to expedite that care. This is a complex question to answer, and was outside the scope of this study. An article by the Society for Academic Emergency Medicine Emergency Department Crowding Task Force discussed the negative impact crowding has on ED patient care and suggests that improving throughput would improve outcomes. ${ }^{17}$ Studies have found that NPs are able to practise as well as residents. ${ }^{18}$ Moreover, many studies have found that high levels of patient satisfaction are achieved with these health care workers. ${ }^{19,20}$ Given the multiple variables involved, it may be impossible to objectively prove that patient care is either better or worse specifically because of PAs or NPs.

\section{Limitations}

The small and retrospective nature of this study carries many potential limitations. The data collection was limited to a 14-day period for each of the baseline and follow-up periods. Such a study is unable to account for seasonal variation or secular trends including bed availability, hospital administrative changes that might have occurred or varying staffing levels due to recruitment. All 6 sites were medium-sized community hospitals; therefore generalization of these results to other hospital sizes may not be valid. Further studies are required to assess the impact on patient flow on a larger scale and in different provinces.

\section{CONCLUSION}

The addition of PAs or NPs to the ED team can improve patient flow in medium-sized community hospital EDs. Given the ongoing shortage of physicians, use of alternative health care providers should be considered. These results require validation, as their generalizability to other locations or types of EDs is not known.

Competing interests: The authors do not have any financial or other conflicts of interest related to this submission. Dr. Tepper is the Assistant Deputy Minister for the Ministry of Health and Long-term Care in the Health Human Resources Strategy Division. A branch of the Ministry under Dr. Tepper's division initiated the project.

\section{REFERENCES}

1. Canadian Institute for Health Information. Understanding emergency department wait times: who is using the emergency departments and how long are they waiting? Ottawa $(\mathrm{ON})$ : The Institute; 2005. Available: http://dsp-psd.pwgsc.gc.ca/Collection /H118-31-2005E.pdf (accessed 2009 Jul 28).

2. Beveridge R, Clarke B, Janes L, et al. Canadian Emergency Department Triage and Acuity Scale: implementation guidelines. CJEM 1999;1(3 suppl).

3. Physician Hospital Care Committee. Improving access to emergency care: addressing system issues. The Committee; 2006. Available: www.health.gov.on.ca/english/public/pub/ministry _reports/improving_access/improving_access.pdf (accessed 2009 Jul 28).

4. Mohsin M, Forero R, Ieraci S, et al. A population follow-up study of patients who left an emergency department without being seen by a medical officer. Emerg Med J 2007;24:175-9.

5. Weiss SJ, Arndahl J, Ernst AA, et al. Development of a site sampling form for evaluation of ED overcrowding. Med Sci Monit 2002;8:CR549-53.

6. Bond K, Ospina MB, Blitz S, et al. Frequency, determinants, and impact of overcrowding in emergency departments in Canada: a national survey. Healthc $Q$ 2007;10:32-40.

7. Defining the PA role in Ontario. Ontario physician assistant scope of practice statement and Ontario physician assistant 
competency profile. Toronto (ON): Health Force Ontario; 2007. Available: www.healthforceontario.ca/upload/en /work/defining\%20the\%20role\%20of\% 20physician\%20assi stant $\% 20$ scope $\% 20$ of $\% 20$ practice $\% 20$ and $\% 20$ competencies $\%$ 20document_\%20may\%209\%202007.pdf (accessed 2009 Jul 28).

8. College of Nurses of Ontario. Nurse practitioners standards of care. Toronto (ON): The College; 2008. Available: www.cno.org/docs/prac/41038_StrdRnec.pdf (accessed 2009 Jul 28).

9. Barr M, Johnston D, McConnell D. Patient satisfaction with a new nurse practitioner service. Accid Emerg Nurs 2000;8: 144-7.

10. Byrne G, Richardson M, Brunsdon J, et al. An evaluation of the care of patients with minor injuries in emergency settings. Accid Emerg Nurs 2000;8:101-9.

11. Rogers T, Ross N, Spooner D. Evaluation of a 'See and Treat' pilot study introduced to an emergency department. Accid Emerg Nurs 2004;12:24-7.

12. Sakr M, Kendall R, Angus J, et al. Emergency nurse practitioners: a three-part study in clinical and cost effectiveness. Emerg Med J 2003;20:158-63.

13. Sanchez M, Smally AJ, Grant RJ, et al. Effects off a fast-track area on emergency department performance. 7 Emerg Med 2006;31:117-20.

14. Rodi SW, Graw MV, Orsini CM. Evaluation of a fast track unit: alignment of resources and demand results in improved satisfaction and decreased length of stay for emergency department patients. Qual Manag Health Care 2006;15:163-70.

15. Thrasher C, Purc-Stephenson R. Patient satisfaction with nurse practitioner care in emergency departments in Canada. J Am Acad Nurse Pract 2008;20:231-7.

16. Ardagh M, Richardson S. Emergency department overcrowding — Can we fix it? N Z Med J 2004;117:U774.

17. Bernstein SL, Aronsky D, Duseja R, et al. The effect of emergency department crowding on clinically oriented outcomes. Acad Emerg Med 2009;16:1-10.

18. Rudy EB, Davidson LJ, Daly B, et al. Care activities and outcomes of patients cared for by acute care nurse practitioners, physician assistants, and resident physicians: a comparison. Am 7 Crit Care 1998;7:267-81.

19. Counselman FL, Graffeo CA, Hill JT. Patient satisfaction with physician assistants (PAs) in an ED fast track. Am J Emerg Med 2000;18:661-5.

20. Venning P, Durie A, Roland M, et al. Randomized controlled trials comparing cost effectiveness of general practitioners and nurse practitioners in primary care. BMJ 2000; 320:1048-53.

Correspondence to: Dr. James Ducharme, 9-6400 Millcreek Dr., Mississauga ON L5N 3E7; jducharme@medemerg.com

\section{À LA RECHERCHE D'UN NOUVEL EMPLOI?}

En version imprimée et en ligne, la meilleure façon de placer une annonce ou de trouver un emploi en médecine d'urgence dans un contexte universitaire, administratif ou communautaire est par le biais du Journal canadien de la médecine d'urgence.

Version imprimée du JCMU : la date butoir pour placer une annonce dans la version imprimée du JCMU est un mois avant la date de parution de la revue. Pour connaître les tarifs pour les annonces et obtenir de plus amples renseignements, veuillez communiquer avec Beverly Kirkpatrick ou Deborah Rodd des Publications de l'AMC (800 663-7336 ou 613 731-8610 x2127 ou x2314, advertising@cma.ca).
À LA RECHERCHE D'UN MÉDECIN D'URGENCE?

PRIME SPÉCIALE : Les médecins qui placeront une annonce dans la version imprimée du JCMU recevront sans frais un espace pour leur annonce sur le site web de l'ACMU (www.caep.ca).

Site web de l'ACMU seulement : Pour placer une annonce sur le site web de l'ACMU seulement, le coût est de 100 \$ par mois et l'annonce peut être placée en tout temps. Veuillez communiquer avec le siège social de l'ACMU pour les annonces sur le site web seulement (800 463-1158 ou advertising @caep.ca). 